\title{
Preovulatory follicle development and ovulation in the brushtail possum (Trichosurus vulpecula) monitored by repeated laparoscopy
}

\author{
J. L. Crawford ${ }^{1,2}$, G. H. Shackell ${ }^{1,2}$, E. G. Thompson ${ }^{1}$, B. J. McLeod ${ }^{1,2}$ \\ and P. R. Hurst ${ }^{2}$ \\ ${ }^{1}$ AgResearch Invermay Agricultural Centre, Private Bag 50034, Mosgiel, New Zealand; and \\ ${ }^{2}$ Department of Anatomy and Structural Biology, University of Otago, New Zealand
}

\begin{abstract}
The common method for synchronizing oestrus in brushtail possums is by removal of their pouch young (RPY). However, there is little information on the ovarian response to this treatment, the timing and incidence of ovulation is poorly defined, and methods of identifying oestrus are unreliable. In this study, the development of preovulatory follicles, ovulation and reproductive tract changes following RPY were monitored by repeated laparoscopic observation. A total of 120 adult female possums underwent laparoscopy at intervals of 1-4 days over the period from 0 to 21 days after RPY. Tissue was collected from a further 30 animals for correlative histology of ovarian structures, and to quantify changes in reproductive tract organs. Only 80 of 120 animals ovulated, and the time of ovulation ranged from 7 to 18 days following RPY. In most animals, enlargement of vaginal cul-de-sac and uterine tissue occurred within 10 days. Correlative histology supported the macroscopic classification of ovarian structures, and healthy and atretic follicles could be identified by laparoscopy. Vaginal smears and plasma progesterone concentrations verified the occurrence of ovulation as observed by laparoscopy. A 'presumptive' preovulatory follicle, first identifiable approximately 5 days before ovulation, was recorded in all animals that ovulated and in none that failed to ovulate. Changes to its surface morphology indicated impending ovulation. This study has enabled the day of ovulation to be identified accurately for the first time in this species. It has also shown that there is wide variation in follicle development, lack of synchrony in the time of ovulation in the brushtail possum, and that some animals fail to ovulate following RPY.
\end{abstract}

\section{Introduction}

In New Zealand, the number of brushtail possums (Trichosurus vulpecula) has escalated following their introduction in the 1830 s, to an estimated current population of $60-70$ million. Since there are no natural predators, attempts have been made to develop methods of biological control through suppression of reproduction. However, progress has been hampered by difficulties in monitoring the oestrous cycle and ovulation in this marsupial.

The female brushtail possum is monovular (Tyndale-Biscoe, 1955) and seasonally polyoestrous (Tyndale-Biscoe, 1955; Lyne et al., 1959; Pilton and Sharman, 1962). The oestrous cycle lasts for 24-26 days (Hartman, 1917; Tyndale-Biscoe, 1955), with a follicular phase of 8-10 days and a luteal phase of 16-18 days. Gross anatomy of the female genital tract includes the cul-de-sac which consists of a median vaginal canal with two lateral canals attached dorsolaterally on either side. Two uteri, each with a separate cervix, extend from the anterior end of the cul-de-sac and lead to the Fallopian tubes and adjoining ovaries (Hartman, 1917).

Revised manuscript received 23 January 1997
Removal of pouch young (RPY) has been the traditional method of inducing and synchronizing oestrus and ovulation in possums (Pilton and Sharman, 1962; Tyndale-Biscoe and Renfree, 1987; Rodger and Mate, 1988), and animals reportedly return to oestrus 4-10 days later. The day of oestrus following RPY has often been estimated by the appearance of cornified epithelial cells in vaginal smears (Hartman, 1917; Pilton and Sharman, 1962), using an approach similar to that used in many eutherian species (for example Restall, 1966; Breed, 1967). However, detection of oestrus using vaginal smears can be imprecise. It is often necessary to take frequent samples to determine when oestrus is approaching in the possum, because errors in estimation can arise if the first appearance of epithelial cells is not detected, or if the appearance of leukocytes is delayed (Tyndale-Biscoe, 1984). Even then, the precise day of oestrus can often be assigned only in retrospect. A more accurate method for determining reproductive status and also of detecting the time of ovulation would be to monitor changes in the reproductive tract and the ovary by direct observation (for example ultrasonography or laparoscopy).

A laparoscopic study of repeated in situ observations of the reproductive tract and ovaries in the female brushtail possum 
is described. This procedure allows gross morphological changes following RPY to be monitored in individual animals. The aim was to visualize the development of the 'presumptive' preovulatory follicle during the periovulatory period and to determine the day of ovulation. Correlative histology was undertaken to confirm the identity of ovarian structures that had been defined by laparoscopic observation. Vaginal smears and plasma progesterone profiles were monitored in some animals to correlate with the laparoscopic observations.

\section{Materials and Methods}

\section{Animals and management}

Adult female brushtail possums (Trichosurus vulpecula) were captured live in the Otago region $\left(45^{\circ} \mathrm{S}\right)$ between $18 \mathrm{April}-24$ August 1995 and 15 March - 8 August 1996. All animals were housed in the AgResearch Invermay possum facility under a group housing system (McLeod et al., 1997). A mixed ad libitum diet of fresh fruit, bread and cereal-based pellets was provided, along with selective browsing of Pinus radiata branches. Fresh water was always available.

All experimental procedures were approved by the $\mathrm{Ag}$ Research Invermay Animal Ethics Committee under the Animal Protection (Codes of Ethical Conduct) Regulations 1987.

\section{Experimental design}

Changes in follicle populations on the surface of the ovary, and in gross morphology of the reproductive tract, were monitored in 150 adult female possums, all of which had pouch young present at the start of the experiment. Preovulatory follicle development and changes in reproductive tract morphology following removal of pouch young (RPY) were determined by repeated laparoscopy in 140 of these animals in the early (April 1996, $n=30$ ), mid- (June 1995, $n=18$; July $1995, n=20$; June 1996, $n=26$ ) or late (August 1995, $n=18$; August 1996, $n=28$ ) breeding season. Each animal underwent a maximum of eight laparoscopic examinations, carried out at intervals of 1-4 days over the period from 0 to 21 days after RPY. Within this group, 120 of 140 animals underwent laparoscopy for $\geq 20$ days following RPY and 14 of these animals were killed after ovulation for tissue collection during the early and late luteal phase of the oestrous cycle (details given below). The remaining 20 animals were killed for tissue collection during the follicular phase before the expected time of ovulation. A further ten possums were killed for tissue collection on the day of RPY and were not subjected to laparoscopy.

A subset of eight animals from the June 1996 replicate underwent laparoscopic examination in conjunction with the collection of vaginal smears and blood samples. Vaginal smears were collected daily from day 0 to day 20 after RPY, and blood samples were taken daily for 7 days from the time of RPY and then every second day for two weeks.

At completion of the period of laparoscopic examination, or at specific times of tissue collection, animals were killed by intra-cardiac administration of Euthal $(4-8 \mathrm{ml} ; 170 \mathrm{mg}$ sodium pentobarbitone $\mathrm{ml}^{-1}, 25 \mathrm{mg}$ sodium phenytoin $\mathrm{ml}^{-1}$; Delta Veterinary Laboratories Pty Ltd, Hornsby, NSW).

\section{Laparoscopy procedure}

Anaesthesia was induced and maintained by halothane inhalation (Fluothane; ICI New Zealand Ltd, Lower Hutt). Animals were transferred to a custom-designed laparoscopy cradle and restrained in a dorsally recumbent position at an angle of $40-50^{\circ}$ with the head end lower. The area between the pouch and sternum on either side of the mid-ventral line was clipped free of fur and scrubbed with isopropyl alcohol. As a prophylactic measure against infection, an injection of antibiotic (Propen S; $1 \mathrm{ml}$ containing $150 \mathrm{mg}$ procaine penicillin, $141.5 \mathrm{mg}$ benethamine penicillin and $250 \mathrm{mg}$ dihydrostreptomycin; Pitman-Moore Animal Health Ltd, Palmerston North) was administered intramuscularly. Local anaesthetic (lignocaine hydrochloride BP 2\% w/v; Techvet Laboratories Ltd, Auckland) was administered subcutaneously as a precautionary measure at two sites $10-20 \mathrm{~mm}$ on either side of the mid-ventral line anterior to the pouch. A small incision was made at the injection sites to facilitate puncturing of the abdominal wall with the endoscope trochar or with a manipulating probe ( $2 \mathrm{~mm}$ diameter). A trochar and cannula were inserted and the abdominal cavity insufflated with air. The trochar was removed and replaced with the endoscope $(4 \mathrm{~mm}$ diameter; Karl Storz GmbH \& Co., Tuttlingen).

Morphology of the cul-de-sac and uteri were assessed subjectively for changes in size, degree of vascularization, colour and swelling (defined as an increase in cross-sectional diameter of tissue due to either thickening of tissue walls and/or distention with mucus). The size of ovarian follicles ( $>1 \mathrm{~mm}$ ) was estimated by comparing their diameter with the open jaws of the manipulating forceps $(6 \mathrm{~mm})$. The reproductive tract and viscera were manoeuvered using a pair of manipulating forceps.

After completion of laparoscopic examination the incisions were closed by a single suture (coated Vicryl 2-0, Ethicon Incorporated, Somerville, NJ) and dusted with antibiotic powder (Negasunt; Bayer New Zealand Ltd, Petone). These incision sites were re-used for repeat laparoscopies on subsequent days of observation.

\section{Laparoscopic analysis of follicular development}

At laparoscopy, follicles were defined as healthy if they were transparent and the follicular fluid was clear, or as atretic if they were opaque and milky white in colour. Progressive changes in colour from white to a distinctive dark brown were considered to be associated with ongoing stages of atresia. Presumptive preovulatory follicles were identified by their large size (3-5 mm) and abundant vascularization, and the extent to which they protruded above the surface of the ovary. Corpora lutea were large $(4-6 \mathrm{~mm})$, highly vascularized, protruding structures that had a distinct stigma. These structures changed temporally in colour from pink to bright red to white. Identification of a corpus albicans was based on successive observations of a luteal structure that progressively diminished in size, whitened in colour and decreased in degree of vascularization. These structures always occurred at the site where a corpus luteum had previously been observed. 


\section{Blood sampling and collection of vaginal smears}

Blood samples ( $1 \mathrm{ml}$ ) were collected from anaesthetized animals (anaesthesia was induced and maintained by halothane inhalation) by jugular venepuncture using a $26 \mathrm{G}$ needle. The samples were centrifuged immediately at $1000 \mathrm{~g}$ for $15 \mathrm{~min}$ and plasma was withdrawn and stored at $-20^{\circ} \mathrm{C}$ for analysis by radioimmunoassay.

Vaginal smears were collected as described by Curlewis $e t$ al. (1985). A saline-moistened cotton bud was passed through a glass speculum that had been inserted into the vaginal opening of the possum, rotated gently to recover material from the cranial urogenital sinus, and removed before the speculum to prevent urine contamination. The cotton bud was smeared on to a clean glass slide, which was air dried and fixed in $70 \%$ ethanol for $1 \mathrm{~min}$. Once dry, slides were stained with methylene blue ( $1 \%$ methylene blue, $1 \%$ azur II, $1 \%$ borax) and observed with an Olympus Provis light microscope. Prooestrus was identified by a marked increase in the number of cornified epithelial cells and the first day of oestrus was defined as the day on which a large influx of neutrophils appeared in the smear (Pilton and Sharman, 1962).

\section{Tissue collection and preparation}

Immediately after the animals were killed, ovaries were recovered, dissected free of mesenteric tissues and immersionfixed in either Bouin's fixative or $0.1 \mathrm{~mol}$ phosphate buffered $10 \%$ formalin $\mathrm{I}^{-1}$, and transferred to $70 \%$ ethanol $24 \mathrm{~h}$ later. Each ovary was embedded in Technovit resin (Kulzer \& Co $\mathrm{GmbH}$, Bereich Technik) and $3 \mu \mathrm{m}$ sections cut and stained with Toluidine blue. Representative sections were prepared from ovarian structures identified and provisionally classified at the time of laparoscopy (see above). Sections were viewed in an Olympus Provis light microscope and photographed using Ilford FP4 black and white film. These sections included examples of healthy and atretic follicles, a 'presumptive' preovulatory follicle, a corpus luteum and a corpus albicans. Reproductive tracts that were removed for colour photography were photographed using AGFA RS 100 slide film.

Reproductive tract tissue was recovered randomly from 44 animals killed on either the day of RPY $(n=10)$ or at one of four different stages of the oestrous cycle: early follicular stage $(n=5)$, late follicular stage $(n=15)$, early luteal stage $(n=11)$ and the late luteal stage $(n=3)$. These stages were identified by laparoscopic examination. The early follicular stage was defined as the time following RPY but before emergence of the 'presumptive' preovulatory follicle, and late follicular stage when a preovulatory follicle was present. The early and late luteal phases were defined as $\leq 9$ days after ovulation and $>9$ days after ovulation, respectively. Immediately after the animals were killed, the vaginal cul-de-sac and uteri were removed, trimmed and weighed.

\section{Progesterone radioimmunoassay}

The assay used to determine progesterone concentration, described and validated for red deer (Asher, 1990), was adapted and validated for brushtail possum plasma. The antiserum was raised in a rabbit against progesterone-11-BSA conjugate and used at a final dilution of 1:30 000. ${ }^{125}$ I-labelled progesterone (Diagnostics Products Corporation, Los Angeles, CA 90045, USA) was used as the tracer. Standards were prepared by adding progesterone (Sigma Chemical Company) at concentrations of 0.1 to $20 \mathrm{ng} \mathrm{ml}^{-1}$ to charcoal-stripped male possum plasma. Serial dilutions of a high concentration possum plasma sample gave an inhibition curve parallel to that of a serial dilution of synthetic progesterone added to charcoal-stripped male possum plasma.

All samples from every animal were included within a single assay and low, medium and high progesterone controls (progesterone diluted in charcoal-stripped male possum plasma) were included at frequent intervals throughout the assay. $10 \mu \mathrm{l}$ of standard, sample or control was added in duplicate to polypropylene tubes with $50 \mu \mathrm{l}$ PVP buffer $(0.1 \%$ polyvinylpyrollidone, MW 44000 ; in $0.05 \mathrm{~mol}$ phosphate buffer $1^{-1}$ ), $100 \mu \mathrm{l}$ antiserum and $200 \mu \mathrm{l}$ tracer (approximately 10000 c.p.m. per tube). All tubes were vortexed and incubated overnight at $4^{\circ} \mathrm{C}$. On the second day $100 \mu \mathrm{l}$ pre-precipitated (made up on the previous day) sheep anti-rabbit second antibody (1:40) mixed with NRS (1:400) in 4\% PEG/PVP buffer (4\% polyethylene glycol 6000; in $0.1 \%$ PVP buffer) was added to each tube, vortexed and incubated overnight at $4^{\circ} \mathrm{C}$. The next day $1 \mathrm{ml} 8 \%$ PEG/PVP ( $8 \%$ PEG in $0.1 \%$ PVP buffer) was added to all tubes and centrifuged at approximately $2500 \mathrm{~g}$ for $35 \mathrm{~min}$. The supernatant was decanted and the pellet counted in a gamma counter.

The intra-assay coefficients of variation were $7.4 \%$ for the low control $\left(1 \mathrm{ng} \mathrm{ml}^{-1}\right), 6.9 \%$ for the medium control $(5 \mathrm{ng}$ $\mathrm{ml}^{-1}$ ) and $6.5 \%$ for the high control (10 $\mathrm{ng} \mathrm{ml}^{-1}$ ). The sensitivity of the standard curve, calculated as twice the standard deviation of the zero standard, was $0.1 \mathrm{ng} \mathrm{ml}^{-1}$.

\section{Statistical analysis}

The masses of cul-de-sac and uterine tissue at the different stages of the oestrous cycle were log transformed to overcome heterogeneity of variance. Comparison between stages of the oestrous cycle were made using a Student's $t$ test. Means are presented with $95 \%$ confidence intervals.

Counts of the number of animals first observed to undergo an event (day of cul-de-sac enlargement, day of uteri enlargement, day of formation of preovulatory follicle and day of ovulation) were grouped into two-day observation intervals, and fitted with a Generalised Linear Model using the Poisson distribution and Log Link (McCullagh and Nelder, 1989).

\section{Results}

\section{Laparoscopy}

In those animals that underwent laparoscopic observations for $\geq 20$ days, 80 ovulated and 40 did not. Changes in follicle populations and in the reproductive tract in the animals that ovulated are given below.

Early follicular phase (days 0-12). Numerous healthy and atretic follicles ( $1-3 \mathrm{~mm}$ diameter) were present at this stage in Downloaded from Bioscientifica.com at 04/26/2023 04:13:53AM 

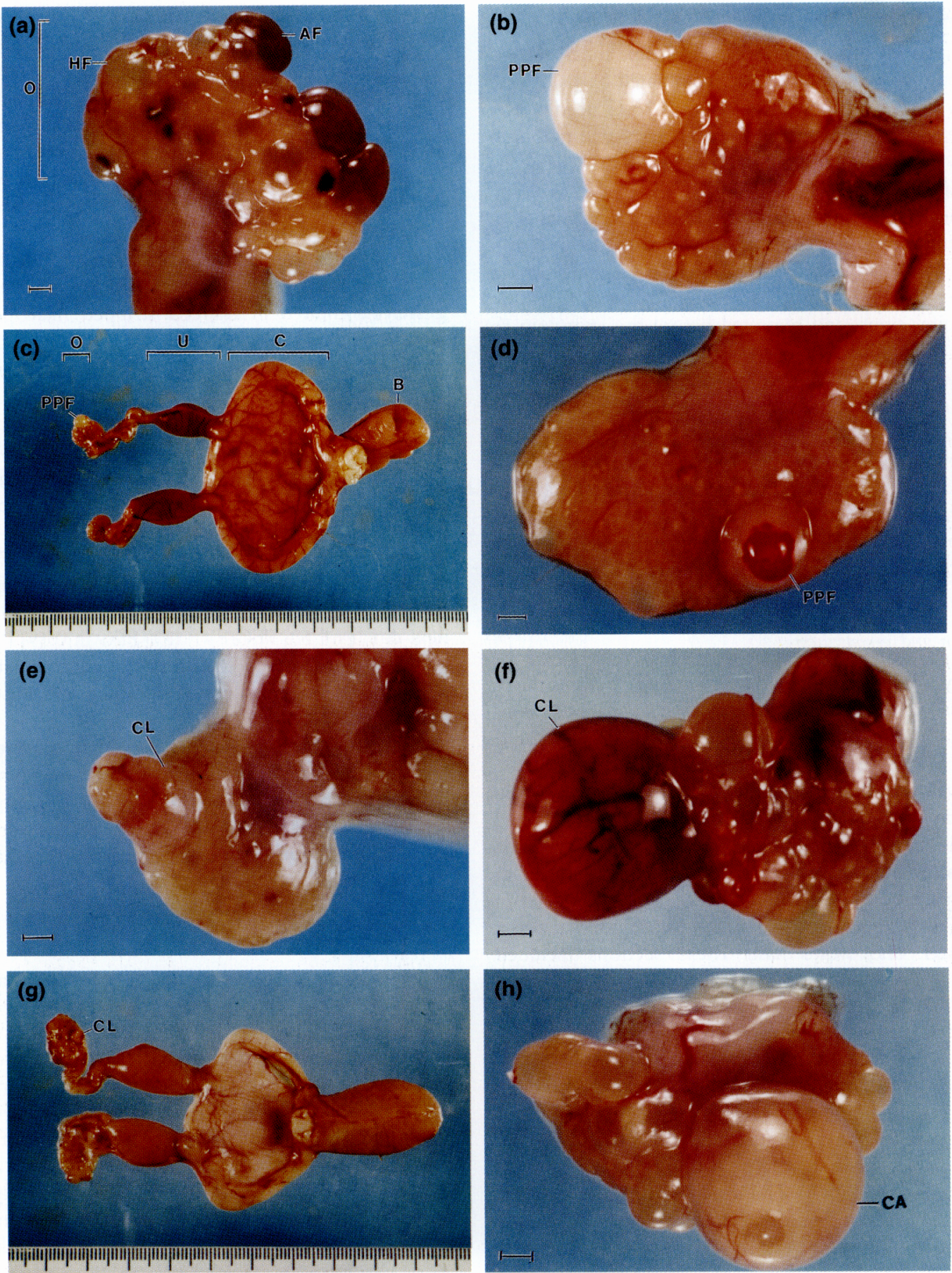

Fig. 1. Ovarian structures and reproductive tracts of the brushtail possum at different stages of the oestrous cycle: (a) ovary $(\mathrm{O})$ in early follicular phase accommodating both healthy (HF) and atretic (AF) follicles; (b) ovary in late follicular phase showing a large, protruding 'presumptive preovulatory follicle (J'l'P): (c) a reproductive tract in late follicular phase. Note large, vascularized cul-de-sac (C) and reddened uteri $U$ ). B indicates the bladder: (d) ovary in late follicular phase. Note the accumulation of blood supply at the apical pole of the "presumptive" preovulatory follicle (PPF); (e) an ovary less than 48 h after ovulation exhibiting a large, opaque corpus luteum (CL): (f) an wary from the early luteal phase ( 9 days after ovulation) displaying a large, vascularized corpus luteum; (g) a reproductive tract in early luteal phase 2 days after ovulation): the uteri have increased in size and the cul-de-sac has regressed in size compared with ict: the an ovary in the late luteal phase. Note the large corpus albicans (CA). Scale bar $=1 \mathrm{~mm}$; (c) and (g) scale ruler in $\mathrm{mm}$. 
all animals (Fig. 1a). Over this period, the cul-de sac and uteri increased progressively in size and the extent of vascularization of these tissues also increased.

Late follicular phase (days 2-13). Presumptive preovulatory follicles were first identified at this time (Fig. Ib). These ranged in diameter from 3 to $6 \mathrm{~mm}$, and their surfaces were highly vascularized. Other small follicles (1-4 mm diameter), both healthy and atretic, were observed in all animals at this time. The cul-de-sac reached a maximum size and became highly vascularized. The uteri also became swollen, vascularized and reddened (Fig. 1c). A distinguishing feature of impending ovulation was the development of a concentrated area of vascularization at the apical pole of the preovulatory follicle (Fig. Id).

Ovulation (days 7-18). This stage was characterized by the presence of a distinct stigma at the ovulation point. Within $48 \mathrm{~h}$ of ovulation, the surface of the developing corpus luteum became opaque and pink (Fig. Ie). Examination of these structures under the microscope revealed the presence of luteal tissue beneath the ovulation point. Cul-de-sac and uterine tissue did not change markedly from earlier observations.

Early luteal phase ( $\leq 9$ days after ovulation; days 8-27). The corpus luteum became vascularized and bright red (Fig. If). During this period, the uteri reached a maximum size and were extremely swollen and highly vascularized. In contrast, the cul-de-sac regressed in size, in comparison with earlier stages (Fig. 1g). Large healthy and atretic follicles were observed on the ovaries of all animals.

Late luteal phase ( $>9$ days after ovulation; days 17-36). At the beginning of this stage the corpus luteum was a large $(4-6 \mathrm{~mm})$, white structure with extensive vascularization. Towards the end of this phase the corpus luteum diminished in size and its vascularization was less distinct (Fig. 1h). Such features were taken to indicate the formation of a corpus albicans. Healthy and atretic follicles were present in all animals.

In the later part of the follicular stage and the early part of the luteal stage, the reproductive tract (cul-de-sac, uteri and fimbria) increased in size markedly, became extensively vascularized and obscured the ovaries from view. Although manipulation of the tract to free the ovaries for viewing was undertaken gently, adhesions formed in 17 out of 140 animals by the completion of the laparoscopic observations.

The interval from RPY to ovulation ranged from 7 to 18 days. In the majority of these animals, the cul-de-sac was first observed to enlarge on day 2 and the uteri on day 2-4 after RPY (Fig. 2). Preovulatory follicle emergence was first observed predominantly between day 6-8 after RPY and the majority of ovulations occurred between day 10 and 14 after RPY.

In those animals that failed to ovulate, a large, healthy follicle ( $\geq 2.5 \mathrm{~mm}$ diameter) was observed during the laparoscopy period in 11 of 40 animals. In all 11 animals, the presence of these follicles was associated with enlargement of the cul-de-sac (Fig. 2). In all instances, these follicles became atretic and the tract regressed in size before completion of the laparoscopic study. In a further 16 animals, a medium-sized, healthy follicle (1.5-2 mm diameter) predominated and in four of these, enlargement of the cul-de-sac and uteri was observed (Fig. 2). Small ( $<1.5 \mathrm{~mm}$ diameter) healthy follicles were observed in the remaining 13 animals, but in these the cul-de-sac and uteri did not increase in size. At no stage did any animal that failed to ovulate have a presumptive preovulatory follicle present.

\section{Histological examination of ovarian tissue}

Those follicles that were macroscopically defined as healthy (Fig. 1a), had evenly distributed layers of granulosa cells and highly vascularized thecal tissue (Fig. 3a). In contrast, follicles that had macroscopically been defined as atretic (Fig. Ia) showed extensive nuclear fragmentation and cell shrinkage and the layers of granulosa cells were irregular (Fig. 3b).

Follicles defined as presumptive preovulatory structures (Fig. 1b) were found to contain a large oocyte, surrounded by an intact zona pellucida (Fig. 3c). The granulosa cells that surrounded the fluid-filled antrum were evenly distributed around the follicle wall.

Structures described as corpora lutea at laparoscopy (Fig. Ie, If) had an extensive mass of luteal cells with an apparent high cytoplasmic: nuclear size ratio (Fig. $3 \mathrm{~d}$ ). These luteal cells were relatively large in diameter, although some smaller luteal cells were seen. The cytoplasm of both large and small luteal cells was characterized by extensive vacuolation.

Corpus albicans (macroscopic appearance shown in Fig. 1h) contained extensive areas of cell debris and degenerating luteal tissue. Strands of fibrous connective tissue were interspersed between the areas of cell debris and luteal tissue (Fig. 3e). No evidence of vascularization was seen.

\section{Changes in uterine and cul-de-sac tissue masses}

Mean masses of reproductive tract tissue recovered from those animals killed at predetermined times during the follicular and luteal phase of the oestrous cycle are shown (Fig. 4). Mean cul-de-sac wet mass (expressed as a proportion of body weight) increased 5.6 times (confidence interval 3.48-9.04) from the early follicular stage to reach a maximum mass during the late follicular stage. In the early luteal stage, this tissue decreased in mass significantly (1.9 times, confidence interval 1.28-2.70). Mean uterus wet mass (in proportion to body weight) increased steadily during the follicular stage to peak at a maximum mass during the early luteal phase (five times the wet mass during the early follicular stage, confidence interval $3.59-6.82$ ).

\section{Plasma concentration of progesterone and vaginal smear cytology}

Four of the eight animals from which blood samples and vaginal smears were collected ovulated following RPY and four did not. In all eight animals progesterone concentrations were low (below the sensitivity of the assay, $0.1 \mathrm{ng} \mathrm{mI}^{-1}$ ) at the time of RPY. In those animals that ovulated, plasma concentrations of progesterone increased above the sensitivity of the assay $\left(0.78-1.99 \mathrm{ng} \mathrm{ml}^{-1}\right)$ within 2 days of ovulation and rose 


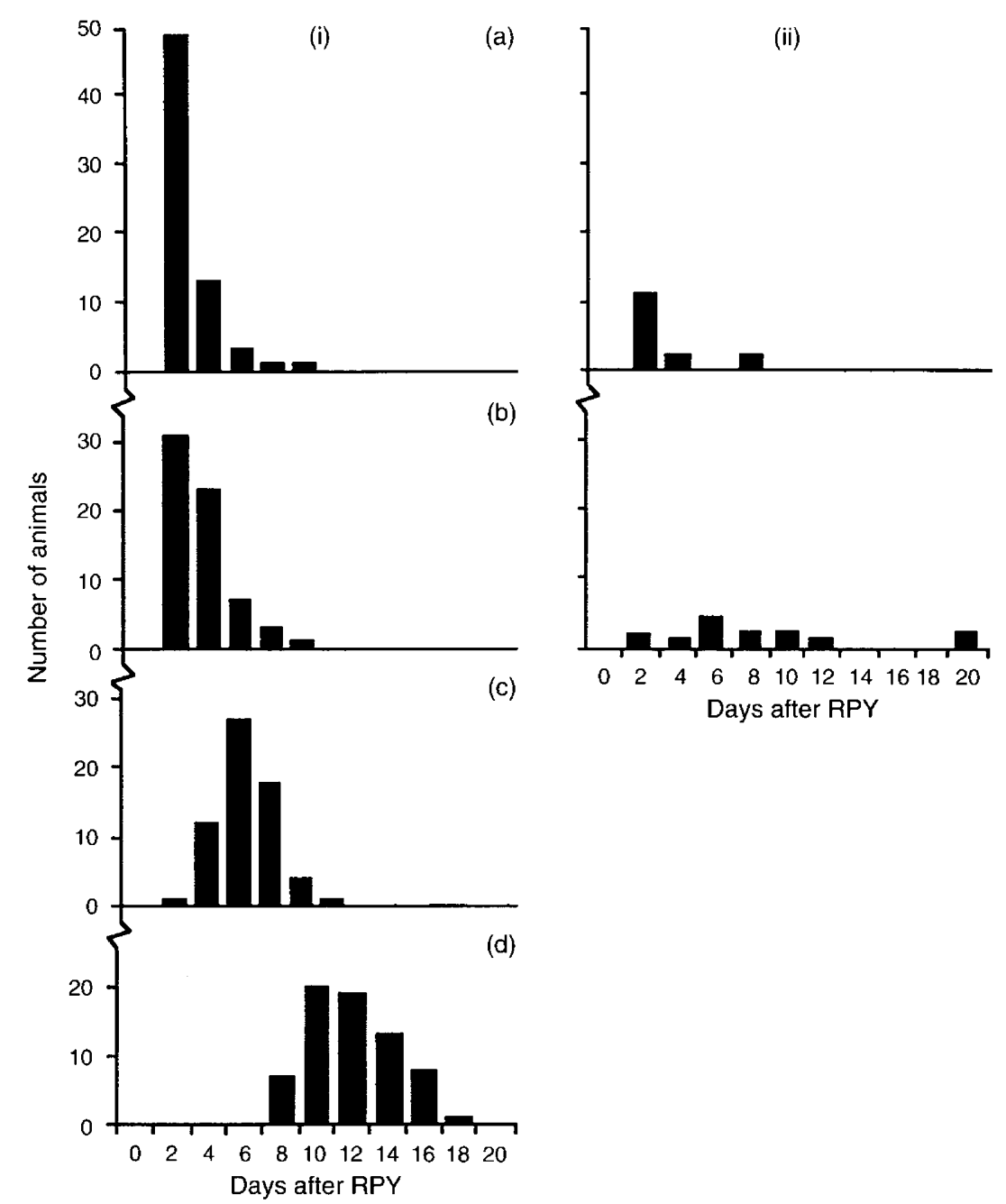

Fig. 2. The day of first observation of (a) an enlarged cul-de-sac, (b) enlarged uteri, (c) a preovulatory follicle and (d) ovulation in female brushtail possums following removal of pouch young (RPY) in (i) animals that ovulated and (ii) animals that failed to ovulate. Observations were made by laparoscopy undertaken at intervals of $1-4$ days for $\geq 21$ days after RPY. Animals that failed to ovulate did not have a preovulatory follicle.

markedly thereafter. Plasma concentrations of progesterone remained below the sensitivity of the assay throughout the sampling period in those animals that failed to ovulate (Fig. 5).

Pro-oestrus, as defined by a large influx of cornified epithelial cells in the vaginal smear, was recorded in all four animals that ovulated. This occurred 1-3 days before ovulation and the duration of a pro-oestrous smear ranged from 2 to 4 days. The day of oestrus, as defined by an influx of neutrophils, was detected from 0 to $I$ days after the day of ovulation in the animals that ovulated. Of the four animals that failed to ovulate, one animal exhibited an oestrus-type smear from 9 to 12 days after RPY, although neutrophil invasion (indicative of the day of oestrus) was less intense compared with smears from animals that ovulated.

\section{Discussion}

This laparoscopic study reported here has shown the wide between-animal variation in preovulatory follicle development and time of ovulation, as well as the high incidence of anovulation, that follows RPY in brushtail possums. Previously, ovarian responses to RPY have been estimated indirectly from changes in vaginal smears, or from enlargement of the reproductive tract determined post mortem. The present laparoscopic study has identified short-comings in both of these methods as these changes occurred in some animals that failed to ovulate. In addition, it has demonstrated that repeated laparoscopic observation of the ovaries can accurately identify those animals that will ovulate after RPY, and that the time of ovulation can be predicted within one day.

Repeated laparoscopic observation of the ovaries in the possum enables the presumptive preovulatory follicle to be identified and easily distinguished from other large, healthy or atretic follicles. The growth and regression of follicles (preovulatory and non-ovulatory) has previously been monitored in other species by repeated laparoscopy (for example sheep, Oldham and Lindsay, 1980; deer, Asher et al., 1990, 1992). In these species, it is often difficult to identify the follicle that is 

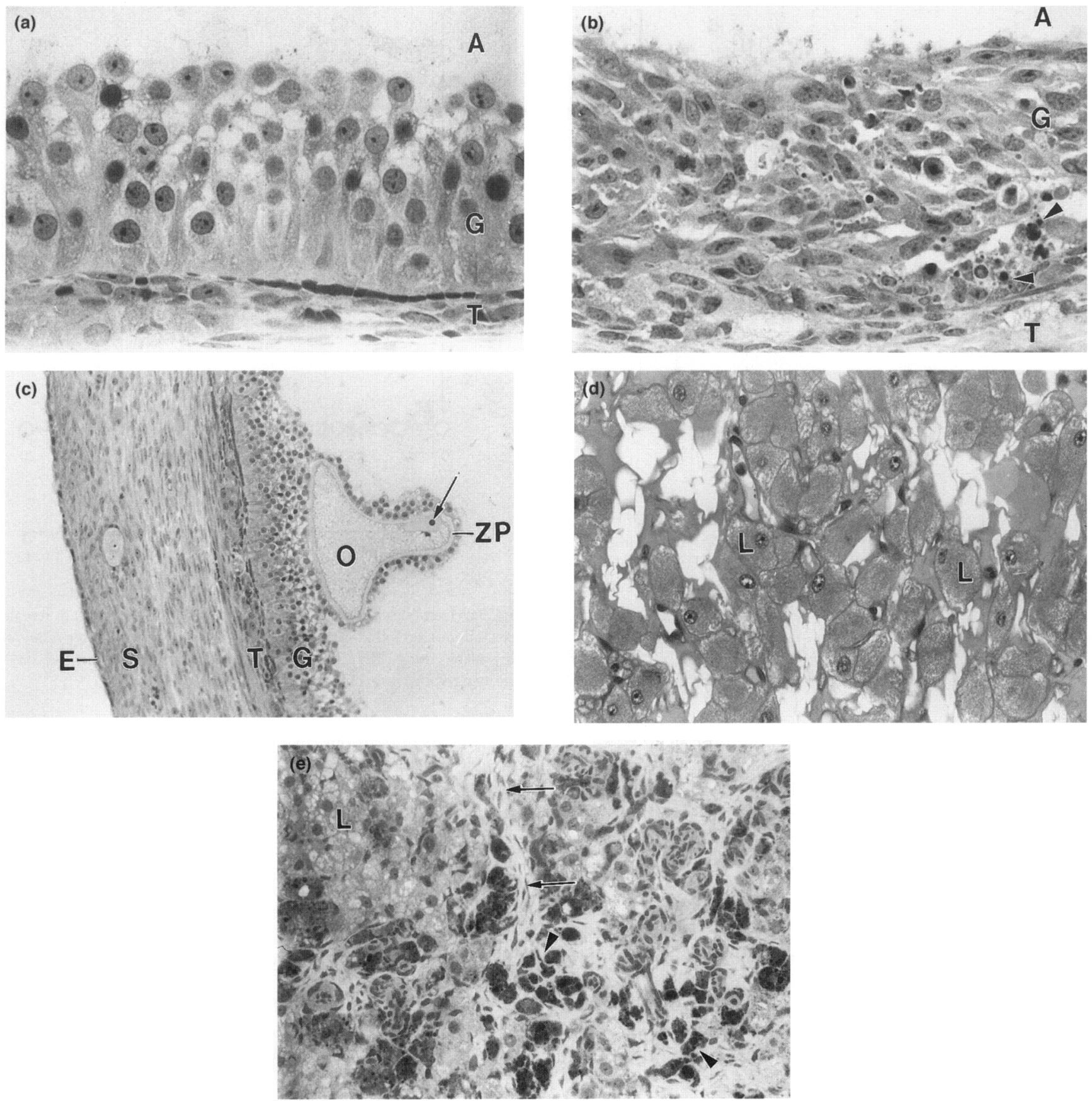

Fig. 3. Histological sections of possum ovarian structures. (a) Wall of a healthy follicle (see Fig. Ia) showing even distribution of granulosa cells (G) adjacent to a follicle antrum $(A)$. T indicates thecal tissue $(\times 600)$. (b) Wall of an atretic follicle (see Fig. Ia). Note the uneven distribution of granulosa cells and evidence of nuclear fragmentation (indicated by arrowheads) $(\times 600)$. (c) Portion of 'presumptive' preovulatory follicle (see Fig. $1 b)$. Section passes through the oocyte $(\mathrm{O})$ and also through granulosa cells, thecal tissue, ovarian stroma $(S)$ and the ovarian surface epithelium (E). ZP indicates zona pellucida; arrow indicates nucleolus ( $\times 150)$; (d) Section of a corpus luteum: note the large luteal cells (L), exhibiting an apparent high cytoplasmic: nuclear size ratio $(\times 300)$. (e) Section of a corpus albicans; note the areas of heterogeneous cell debris (indicated by arrowheads) and old luteal tissue. Strands of connective tissue are interspersed between these areas (indicated by arrows) ( $\times 300$ ).

destined to ovulate because there may be several candidate follicles of similar size. In contrast, in possums the preovulatory follicle was always identifiable (approximately 5 days before ovulation), and obvious changes in its surface morphology provided reliable indicators of impending ovulation. With the exception of those animals that were killed for recovery of tract tissue late in the follicular phase, ovulation occurred in every animal deemed to have had a preovulatory follicle. Furthermore, in the latter part of this study when the sequence of morphological changes that precede ovulation had been characterized, it was possible to predict the time of ovulation accurately. More than $90 \%$ of animals predicted to ovulate 


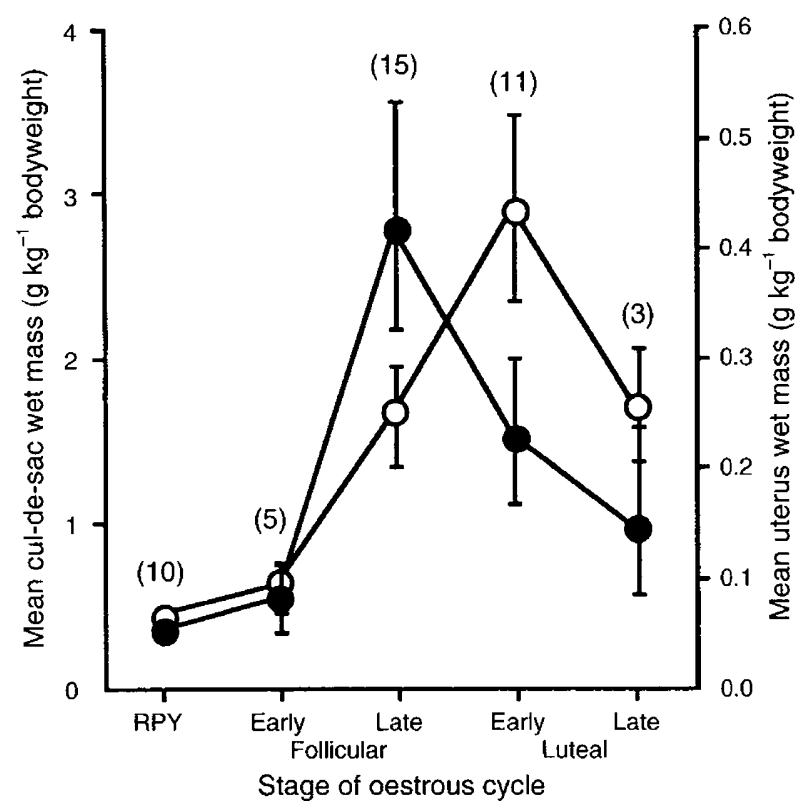

Fig. 4. Mean cul-de-sac (O) and uteri (O) masses $\left(\mathrm{g} \mathrm{kg}^{-1}\right.$ body weight; error bars denote $95 \%$ confidence interval of the mean) in female brushtail possums at the time of removal of pouch young (RPY) and at four different stages of the oestrous cycle. The early and late follicular phases are characterized by the absence or presence of a 'presumptive' preovulatory follicle, respectively. The early and late luteal phases are $\leq 9$ days after ovulation and $>9$ days after ovulation, respectively. Numbers of animals are shown in parentheses.

within $24 \mathrm{~h}$ of a particular laparoscopic observation, did so $(n=24)$. Even though preovulatory follicles were not tagged in any way other than by recording their location on the ovary, there was convincing circumstantial evidence that they eventually ovulated. In all animals with a preovulatory follicle, an ovulation point was later observed at the same site on the same ovary.

The present study has shown that preovulatory follicle development after RPY was asynchronous between animals. Although laparoscopic examination within the first few days after RPY can provide an initial indication of ovarian activity, some caution is necessary, since swelling of the reproductive tract and the presence of follicles greater than $1.5 \mathrm{~mm}$ diameter was observed at this time in possums that subsequently failed to ovulate (15 of 40). It is presumed that growth and atresia of antral follicles (1.5-3 mm diameter) occur throughout the oestrous cycle. Therefore, developing follicles may have been present in some animals, and these follicles presumably would have had the ability to secrete oestrogen at a level sufficient to induce proliferation of the reproductive tract but they failed to ovulate.

It is unlikely that the high incidence of anovulation following RPY in the present study was simply due to laparoscopy and/or anaesthesia. Firstly, the percentage of animals that failed to ovulate in each experimental group ranged from 0 to $85 \%$, and all animals were subjected to similar surgical procedures. Although the effect of halothane on reproductive hormones is unknown in the possum, some animals that were not subjected to halothane also did not return to oestrus after RPY. Ovulation failure has frequently been observed in slaughter
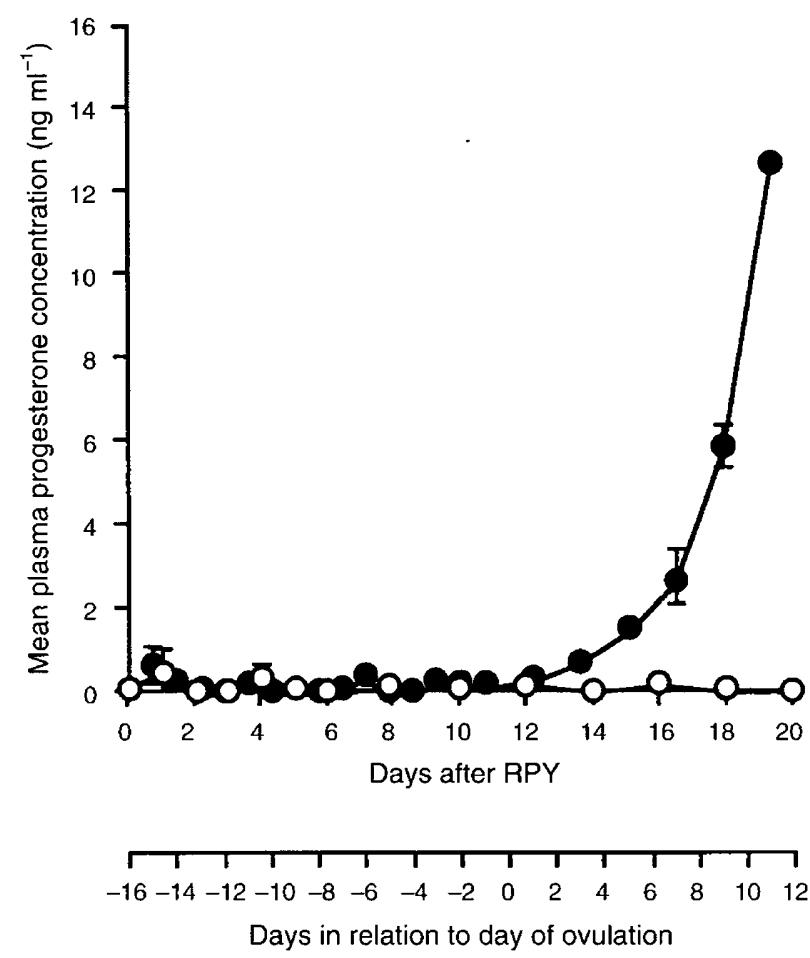

Fig. 5. Mean plasma progesterone concentration ( $\mathrm{ng} \mathrm{ml}^{-1}$ ) in female brushtail possums that did $(O)$ and $\operatorname{did}$ not $(\bigcirc)$ ovulate after removal of pouch young (RPY), in relation to day of ovulation and days following RPY, respectively. Error bars denote SEM.

experiments in both our own (J. L. Crawford and B. J. McLeod, unpublished) and other (D.C. Eckery, personal communication) possum colonies. Halothane anaesthesia has been shown to reduce the pulsatile secretion of $\mathrm{LH}$ in ewes. However, this only occurs during anaesthesia and the pulsatile activity of $\mathrm{LH}$ recovers quickly (Clarke and Doughton, 1983). Animals in this study were subjected to halothane for only 5-10 min per laparoscopic examination, so it is unlikely that follicle development would be affected. Stress imposed by restraint during pro-oestrus has been shown to suppress the preovulatory gonadotrophin surge in rats partially or completely (Roozendaal et al., 1995). This effect is unlikely to have occurred in the present study, as recent trials have shown $100 \%$ incidence of ovulation in possums that underwent an extensive laparoscopic programme that required restraint (J. L. Crawford and B. J. McLeod, unpublished). Other observations of unexpected responses to RPY in possums have also been reported (Curlewis and Stone, 1986). The reasons for failure of ovulation remain to be determined.

The presence of non-ovulatory follicles with the ability to produce oestrogen may explain the difficulties that are often encountered in interpreting cytological changes in vaginal smears that are often used for the detection of oestrus in this species. For example, in the present study one of the four animals in which vaginal smear cytology was monitored and that failed to ovulate, exhibited a smear pattern characteristic of an oestrus state for 4 days, and this coincided with the presence of a large non-ovulatory follicle $(4 \mathrm{~mm})$ and an enlarged cul-de-sac and uteri. Similar observations have been made in the vole, where vaginal mucous cycles may reflect irregular, 
nonovulatory follicular changes (Peterson, 1986). Even though vaginal smears from the four animals that did ovulate were indicative of ovulation, these were not precisely related to the time of ovulation.

Classification of follicle health by gross morphological examination at laparoscopy was supported by histological examination. Follicles defined as healthy $(n=10)$, had evenly distributed granulosa surrounded by a highly vascularized thecal tissue layer, consistent with that seen in normal healthy follicles and there was no evidence of atresia. In contrast, follicles classified as atretic $(n=10)$, had irregular layers of granulosa cells, nuclear fragmentation and cell shrinkage, all of which are characteristic of atresia. Preovulatory follicles $(n=3)$ had histological features of healthy follicles. Although the oocyte in the preovulatory follicle shown is irregular in shape, this was possibly an artefact of fixation or processing.

The observation (both at laparoscopy and from tissue collection) that the maximum size of cul-de-sac tissue coincided with the time of presumptive preovulatory follicle formation, and that the uteri attained their largest size during the early luteal phase, suggests that ovarian steroids are involved. Oestrogens are secreted at high concentrations during the follicular phase and the endocrine status of the luteal phase is dominated by progesterone secreted from the corpus luteum (Curlewis et al., 1985; Karsch et al., 1992). One of the more significant roles of progesterone is to prepare the uterus for pregnancy. Therefore, it would seem appropriate that the maximum size of the possum uterus coincided with the time when fertilization and embryonic development would be initiated. The fact that the cul-de-sac attained its maximum size during the follicular phase raises several possible suggestions for its function, which is yet to be determined. For example, the cul-de-sac and its secretions may play a primary role in sperm storage, selection, transport and/or capacitation. It is of interest to note the changes in reproductive tract tissues that occurred in response to RPY. Enlargement of the cul-de-sac occurred within a few days of RPY, even in a proportion (15/40) of animals that failed to ovulate. There was also a rapid increase in uterine size, especially in those animals that ovulated. When animals were either killed on the day of RPY or laparoscopy was performed at this time, both the cul-de-sac and uteri were at their smallest.

Although laparoscopy offers a rapid and accurate method for determining the day of ovulation in the female possum, this procedure was not without problems. Manipulation of the reproductive tract at laparoscopy was complicated by its size and anatomical position. As the cul-de-sac lies ventral to the ovaries and there was an increase in its size during the follicular phase, it often obscured the ovaries from view. In addition, the fimbria became highly vascularized and enlarged at this time, and gradually surrounded the ovaries. For these reasons, careful use of the manipulating probe was necessary to view the complete surface of the ovary. Adhesions had formed on the reproductive tracts of only 17 animals by completion of the laparoscopic observations.

Removal of pouch young has been the traditional method of inducing and synchronizing oestrus and ovulation in possums (Pilton and Sharman, 1962; Tyndale-Biscoe and Renfree, 1987; Rodger and Mate, 1988). The present study has shown a wide range in the interval from RPY to ovulation and furthermore that some animals fail to ovulate. This study also demonstrated that marked changes in reproductive tract morphology often occur in animals that fail to ovulate. As these changes in the uterus and cul-de-sac are presumably driven by increased oestrogen secretion, they are likely to be accompanied by those changes in vaginal smear cytology that have been used as indicators of oestrus and ovulation. Use of the laparoscope to directly monitor ovarian function can identify follicle development and the specific day of ovulation.

The authors thank P. D. Johnstone for statistical analyses and L. Fenton for technical assistance. The authors are grateful to the MAF Policy division of the New Zealand Ministry of Agriculture and Fisheries for funding this study. J. Crawford was supported by a research fellowship from MAF Policy.

\section{References}

Asher GW (1990) Effect of subcutaneous melatonin implants on the seasonal attainment of puberty in female red deer (Cervus elaphus) Animal Reproduction Science 22 145-159

Asher GW, Fisher MW, Smith JF, Jabbour HN and Morrow CJ (1990) Temporal relationship between the onset of oestrus, the preovulatory LH surge and ovulation in farmed fallow deer, Dama dama. Journal of Reproduction and Fertility 89 761-767

Asher GW, Fisher MW, Jabbour HN, Smith JF, Mulley RC, Morrow CJ, Veldhuizen FA and Langridge M (1992) Relationship between the onset of oestrus, the preovulatory surge in luteinizing hormone and ovulation following oestrous synchronization and superovulation of farmed red deer (Cervus elaphus) Journal of Reproduction and Fertility 96 26I-273

Breed WG (1967) Ovulation in the genus Microtus. Nature 214826

Clarke IJ and Doughton BW (1983) Effect of various anaesthetics on resting plasma concentrations of luteinizing hormone, follicle-stimulating hormone and prolactin in ovariectomized ewes Journal of Endocrinology $\mathbf{9 8}$ $79-89$

Curlewis JD and Stone GM (1986) Reproduction in captive female brushtail possums, Trichosurus vulpecula. Australian Journal of Zoology 34 47-52

Curlewis JD, Axelson M and Stone GM (1985) Identification of the major steroids in ovarian and adrenal venous plasma of the brushtail possum (Trichosurus vulpecula) and changes in the peripheral plasma levels of oestradiol and progesterone during the reproductive cycle journal of Endocrinology 105 53-62

Hartman C (1917) The oestrous cycle in the opossum Biological Bulletin 37 353-42I

Karsch FJ, Moenter SM and Caraty A (1992) The neuroendocrine signal for ovulation Animal Reproductive Science 28 329-334

Lyne AG, Pilton PE and Sharman GB (1959) Estrous cycle, gestation period and parturition in the marsupial Trichosurus vulpecula. Nature $\mathbf{1 8 3} 622-623$

McCullagh P and Nelder JA (1989) In Generalised Linear Models (Second Edn) Chapman and Hall Publishers, London

McLeod BJ, Thompson EG, Crawford IL and Shackell GH (1997) Successful group-housing of wild-caught brushtail possums (Trichosurus vulpecula) Animal Welfare 6 67-76

Oldham CM and Lindsay DR (1980) Laparoscopy in the ewe: a photographic record of the ovarian activity of ewes experiencing normal or abnormal oestrous cycles Animal Reproduction Science 3 119-124

Peterson SL (1986) Age- and hormone-related changes in vaginal smear patterns in the gray-tailed vole, Microtus canicaudus. Journal of Reproduction and Fertility 78 49-56

Pilton PE and Sharman GB (1962) Reproduction in the marsupial Trichosurus vulpecula. Journal of Endocrinology 25 119-136

Restall BJ (1966) Histological observations on the reproductive tract of the ewe Australian Journal of Biological Science 19 673-686

Rodger JC and Mate KE (1988) A PMSG/GnRH method for the superovulation of the monovulatory brush-tailed possum (Trichosurus vulpecula) Journal of Reproduction and Fertility $\mathbf{8 3} 885-891$ 
Roozendaal MM, Swarts HJ, Wiegant VM and Mattheij JA (1995) Effect of restraint stress on the preovulatory luteinizing hormone profile and ovulation in the rat European Journal of Endocrinology 133 347-353

Tyndale-Biscoe $\mathrm{CH}$ (1955) Observations on the reproduction and ecology of the brush-tailed possum, Trichosurus vulpecula Kerr (Marsupialia), in New Zealand Australian Journal of Zoology 3(2) 162-185
Tyndale-Biscoe CH (1984) Reproductive physiology of possums and gliders. In Possums and Gliders pp 79-87 Eds AP Smith and ID Hume. Australia Mammal Society, Sydney

Tyndale-Biscoe CH and Renfree MB (1987) In Reproductive Physiology of Marsupials, pp 55-57 Cambridge University Press, Cambridge 